\title{
Mediciones continuas de carbono negro, monóxido de carbono y dióxido de carbono, durante la temporada seca caliente 2016, en un sitio periurbano de Querétaro, México
}

\section{Continuous measurements of black carbon, carbon monoxide and carbon dioxide during dry warm-season 2016 in a peri-urban area of Queretaro, Mexico}

\author{
${ }^{1,2, *}$ Rafael Liñán Abanto \\ ${ }^{1}$ Telma Castro \\ Oscar Peralta \\ ${ }^{1}$ Luis G. Ruiz Suárez
}

\author{
${ }^{3}$ Dara Salcedo \\ ${ }^{4}$ Giovanni Carabali \\ ${ }^{5}$ Patrick Arnott \\ Guadalupe Paredes Miranda
}

\section{RESUMEN}

En Juriquilla-Querétaro, se midió la concentración en masa de carbono negro (BC), la relación de mezcla de monóxido de carbono (CO) y el dióxido de carbono $\left(\mathrm{CO}_{2}\right)$. Las mediciones se realizaron del 01 de marzo al 30 de abril de 2016. En abril, los valores medios del BC $\left(1.23 \mu \mathrm{g} / \mathrm{m}^{3}\right)$ y CO $(0.30 \mathrm{ppm})$ fueron mayores a sus correspondientes del mes de marzo $\left(1.08 \mu \mathrm{g} / \mathrm{m}^{3}\right.$ y $0.26 \mathrm{ppm}$, respetivamente). El $\mathrm{CO}_{2}$ no sufrió variación en estos meses y mantuvo un valor casi constante de alrededor de $406 \mathrm{ppm}$. Las tendencias diarias del $\mathrm{BC}, \mathrm{CO}$ y $\mathrm{CO}_{2}$ fueron similares, con un máximo pronunciado por la mañana y un mínimo por la tarde. Estos contaminantes alcanzaron su valor máximo alrededor de las 08:00-09:00 Hora Local (HL). Se presentó una buena correlación en el ajuste lineal de $\mathrm{BC}-\mathrm{CO}, \mathrm{BC}-\mathrm{CO}_{2}$ y CO-CO estos contaminantes provendrían de las mismas fuentes. Se observó un efecto fin de semana (valores más bajos del BC, $\mathrm{CO}$ y $\mathrm{CO}_{2}$ el fin de semana en comparación con los de días laborables), principalmente en las horas de máximo tráfico. $\mathrm{Al}$ no existir estudios de mediciones continuas de $\mathrm{BC}, \mathrm{CO}$ y $\mathrm{CO}_{2}$; desarrollamos esta investigación para determinar las tendencias diarias de estos contaminantes y sus correlaciones mutuas en una zona periurbana, en Querétaro.

Palabras clave: Carbono negro, dióxido de carbono, efecto fin de semana, monóxido de carbono.

\section{ABSTRACT}

In Juriquilla-Queretaro, the mass concentration of black carbon (BC), the mixing ratio of carbon monoxide (CO) and carbon dioxide (CO2) were measured. Measurements were made from March 1 to April 30, 2016. In April, the mean values of $\mathrm{BC}(1.23 \mu \mathrm{g} / \mathrm{m} 3)$ and $\mathrm{CO}(0.30 \mathrm{ppm})$ were higher than their corresponding values for March $(1.08 \mu \mathrm{g} / \mathrm{m} 3$ and $0.26 \mathrm{ppm}$, respectively). $\mathrm{CO} 2$ did not variate in these months and maintains almost a stabilized value around $406 \mathrm{ppm}$. The daily trends of $\mathrm{BC}, \mathrm{CO}$, and $\mathrm{CO} 2$ were similar with a pronounced maximum in the morning and a minimum in the afternoon. These pollutants reach a high level around 08:00-09:00 Local Time (LT). A good correlation was estimated in the linear regression of $\mathrm{BC}-\mathrm{CO}, \mathrm{BC}-\mathrm{CO} 2$, and $\mathrm{CO}-\mathrm{CO} 2$, suggesting that these pollutants proceed from the same sources. A weekend effect (lower values of $\mathrm{BC}, \mathrm{CO}$, and $\mathrm{CO} 2$ on the weekend compared to working days) was mainly observed during rush-hour traffic. As there are no studies of continuous measurements of $\mathrm{BC}, \mathrm{CO}$, and $\mathrm{CO}$, this research was conducted to determine the daily trends of these pollutants and their mutual correlations in a peri-urban area of Queretaro.

Keywords: Black carbon, carbon dioxide, weekend effect, carbon monoxide.

\footnotetext{
Centro de Ciencias de la Atmósfera, Universidad Nacional Autónoma de México (UNAM), Circuito Exterior s/n, Ciudad Universitaria, Ciudad de México, 04510, México.

${ }^{2}$ Departamento de Física, Facultad de Ciencias. Universidad Nacional Jorge Basadre Grohmann, Av. Miraflores s/n, Tacna, 23004, Perú.

MDI-Juriquilla, Facultad de Ciencias. Universidad Nacional Autónoma de México, Boulevard Juriquilla 3001, Col. Juriquilla, Querétaro, 76230, México.

${ }^{4}$ Instituto de Geofísica. Universidad Nacional Autónoma de México (UNAM), Circuito Exterior s/n, Ciudad Universitaria, Ciudad de México, 04510, México.

${ }^{5}$ Department of Physics. University of Nevada, 1664 N. Virginia Street, Reno, Nevada, 89557, USA.
}

*Autor de correspondencia: rafael.linian@gmail.com 


\section{INTRODUCCIÓN}

La atmósfera terrestre está compuesta principalmente por gases, pero también contiene materia líquida y sólida en forma de partículas. Las partículas pueden ser sólidas o líquidas, con diámetros entre $\sim 0.002 \mu \mathrm{m}$ y $\sim 100 \mu \mathrm{m}$. El extremo inferior del rango de tamaño no está definido claramente porque no hay un criterio aceptado, en el cual un grupo de moléculas se convierta en una partícula. El extremo superior corresponde al tamaño de llovizna fina o arena muy fina; estas partículas son tan grandes que rápidamente precipitan. Las partículas más importantes para la física y química atmosférica se encuentran en el rango de $0.002 \mu \mathrm{m}$ a $10 \mu \mathrm{m}$ (Finlayson \& Pitts, 2000).

Un aerosol es definido como una suspensión de partículas finas sólidas o líquidas en un gas. Se emiten directamente como partículas (aerosol primario) o se forman en la atmósfera mediante procesos de conversión de gas a partícula (aerosol secundario). La diferencia entre los aerosoles y las partículas radica en que un aerosol incluye tanto las partículas como el gas en el que están suspendidas. Los aerosoles pueden ser de origen natural o antropogénico. (IPCC, 2013; Finlayson \& Pitts, 2000; Seinfeld \& Pandis, 2006).

El Carbono Negro (Black Carbon, BC) es un tipo de material carbonoso que se forma principalmente en llamas durante la combustión incompleta y se emite directamente a la atmósfera. Se encuentra generalmente en aerosoles atmosféricos que contienen otros materiales, muchos de los cuales son emitidos junto con el $\mathrm{BC}$ desde una variedad de fuentes (IPCC, 2013; Bond et al., 2013). También, es emitido en la combustión incompleta de combustibles fósiles, biocombustibles y biomasa (Kondo et al., 2006; Han et al., 2009). El BC tiene muy baja reactividad química en la atmósfera; su proceso de remoción principal a la superficie es a través del depósito húmedo o seco. Similar a los gases de efecto invernadero, como agente de calentamiento climático, el $\mathrm{BC}$ puede influenciar en los procesos de formación de nubes, el derretimiento de la nieve y el albedo.

En la atmósfera, el dióxido de carbono $\left(\mathrm{CO}_{2}\right)$ es el gas traza dominante y es el mayor contribuyente al incremento del forzamiento radiativo. Entre 1750 y 2011, las emisiones antropogénicas de $\mathrm{CO}_{2}$ fueron de $555 \pm 85$ Petagramos $\left(\mathrm{Pg}=10^{15}\right.$ gramos). Estas emisiones provienen principalmente de la combustión de combustibles fósiles $(375 \pm 30 \mathrm{Pg}$ ) y las derivadas de cambios de uso de suelos (180 \pm 80 Pg) (IPCC, 2013). Los océanos y ecosistemas terrestres actúan como sumideros; además, almacenan aproximadamente la mitad de las emisiones de $\mathrm{CO}_{2}$, la otra mitad $(240 \pm 10 \mathrm{Pg})$ permanecen en la atmósfera. La relación de mezcla de $\mathrm{CO}_{2}$ troposférico ha aumentado de 278 ppm (en 1750) a 390.5 ppm (en 2011) (Ballantine et al., 2012; IPCC, 2013). Las mediciones de $\mathrm{CO}_{2}$ exhiben un ciclo estacional. En el hemisferio norte, durante la primavera y el verano, el $\mathrm{CO}_{2}$ atmosférico disminuye a medida que el carbono se incorpora a las plantas frondosas; mientras que en el otoño e invierno (octubre a febrero) se incrementa los niveles de $\mathrm{CO}_{2}$, por un dominio de la respiración y descomposición de las plantas (Seinfeld \& Pandis, 2006).

El monóxido de carbono atmosférico (CO) es producido por la combustión incompleta de combustibles fósiles. En las grandes zonas urbanas de los países desarrollados, la mayor fuente son los escapes de los vehículos ligeros a gasolina (light duty gasoline vehicles, LDGVs). Las fuentes naturales de $\mathrm{CO}$ incluyen $\mathrm{CO}$ de la quema de biomasa y la oxidación de material orgánico, tal como el metano e isopreno, $\mathrm{CO}$ de procesos biológicos en suelos, $\mathrm{CO}$ de vegetación y termitas, y CO del océano (Finlayson \& Pitts., 2000). Al CO no se le considera como un gas de efecto invernadero; sin embargo, es el contaminante primario más abundante en la atmosfera. Según la Norma Oficial Mexicana, el límite máximo permisible (LMP) del CO es 11 ppm, promedio móvil de 8 horas. Por otro lado, la Agencia de Protección Ambiental de los Estados Unidos (Environmental Protection Agency, EPA) establece como LMP 35 ppm, promedio máximo de una hora; y 9 ppm, promedio máximo de ocho horas (Olivares, 2016).

El objetivo general de este trabajo fue determinar la tendencia diaria y mensual de las concentraciones del $\mathrm{BC}, \mathrm{CO}$ y $\mathrm{CO}_{2}$; además, establecer la influencia de los parámetros meteorológicos sobre estos contaminantes, en un sitio en pleno desarrollo urbanístico (zona periurbana) en Querétaro. 


\section{MATERIAL Y MÉTODOS}

\section{Sitio de medición: Juriquilla-Querétaro}

El observatorio de la RUOA en JuriquillaQuerétaro (JQRO) se encuentra en el campus de la UNAM en Juriquilla, ubicado en el límite norte del Área Metropolitana de Querétaro $\left(20^{\circ} 42^{\prime} 11^{\prime \prime}\right.$ $\mathrm{N}$; 100²6'50" O, $1893 \mathrm{~m} \mathrm{s.} \mathrm{n.} \mathrm{m).} \mathrm{La} \mathrm{toma} \mathrm{de}$ muestras de aire se realizó aproximadamente a 15 m s. n. m. Este campus está rodeado de áreas residenciales y comerciales, adyacentes a una carretera con tránsito mixto.

Querétaro tiene 1.2 millones de habitantes (aproximadamente el $45 \%$ de la población del Estado) y está ubicada a $220 \mathrm{~km}$ al noroeste de la Ciudad de México. Su clima predominante es templado semiseco, con un rango de temperatura de $12{ }^{\circ} \mathrm{C}$ a $20^{\circ} \mathrm{C}$ y precipitación entre $500 \mathrm{~mm}$ y $700 \mathrm{~mm}$ (INEGI, 2018).

\section{Métodos e instrumentos de medición}

Para el análisis de las variaciones mensuales de concentración en masa de $\mathrm{BC}$, relación de mezcla de $\mathrm{CO}$ y $\mathrm{CO}_{2}$, y los parámetros meteorológicos (humedad relativa, temperatura y velocidad del viento) se obtuvieron sus valores horarios promediando las mediciones registradas en minutos o segundos. Posteriormente, los datos horarios se agruparon por meses y la temporada seca caliente, que va de marzo a abril en la región central de la republica de los Estados Unidos Mexicanos (Jáuregui, 2002; de Foy et al., 2005; Bravo et al., 2014).

El BC se midió in situ y en forma continua, utilizando un Extinciómetro Fotoacústico (Photoacoustic Extinctiometer, PAX, DMT Inc.). Este instrumento realiza mediciones directas de las propiedades ópticas de las partículas. Utiliza un diodo láser modulado a $1500 \mathrm{~Hz}$, a una longitud de onda de $870 \mathrm{~nm}$ (específica para partículas de carbono negro. Un flujo nominal de muestras de aerosol de $1 \mathrm{~L} /$ min pasa a través del PAX utilizando una bomba de vacío interna controlada por dos orificios críticos. Las mediciones de absorción utilizan tecnología fotoacústica (Arnott et al., 1999, 2000). Un rayo láser dirigido a través de la corriente de aerosol se modula a la frecuencia de resonancia de la cámara acústica. Las partículas absorbentes se calientan y transfieren rápidamente el calor al aire circundante. El calentamiento periódico produce ondas de presión que pueden detectarse con un micrófono sensible. El coeficiente de absorción es directamente proporcional a la presión detectada por el micrófono. La concentración de masa de carbono negro (BC) se obtiene a partir del coeficiente de absorción.

Un analizador que utiliza la técnica de la Espectroscopia de Cavidad "Ring-Down" (Cavity Ring-Down Spectroscopy, CRDS, G2401, Picarro Inc.) fue utilizado para realizar mediciones continuas y simultaneas del $\mathrm{CO}$ y $\mathrm{CO}_{2}$ a nivel de trazas. Este espectrómetro de cavidad tipo "ring-down" se compone básicamente de: a) un láser; b) una cavidad óptica resonante con dos o más espejos de alta reflectividad; y, c) un fotodetector. La luz del láser se dirige a la pequeña cavidad y cuando la acumulación de luz en la cavidad (con la muestra) alcanza la intensidad requerida, el láser se apaga. El fotodetector detecta la pequeña cantidad de luz que se filtra a través de uno de los espejos para producir una señal que es directamente proporcional a la intensidad en la cavidad. La intensidad de la luz en la cavidad decae exponencialmente con una constante de tiempo, $\tau$, que depende de las pérdidas debidas a los espejos de la cavidad y la absorción y dispersión de la muestra que se está midiendo. La absorbancia óptica de la muestra está determinada por la tasa de disipación de luz en la cavidad óptica. De esta manera se mide las concentraciones o relaciones isotópicas de un gas de interés (Crosson, 2008; Chen et al., 2010).

\section{Análisis de datos}

Para el análisis estadístico de las mediciones de los contaminantes $\mathrm{BC}, \mathrm{CO}$ y $\mathrm{CO}_{2}$, durante los meses de marzo y abril, utilizamos sus valores registrados en minutos. Los datos horarios de $\mathrm{BC}$, $\mathrm{CO}$ y $\mathrm{CO}_{2}$ se obtuvieron promediando las mediciones registradas (en segundos o minutos) se agruparon de dos maneras: (1) para toda la temporada seca caliente y (2) por meses (marzo y abril). Similarmente, se agruparon los promedios horarios de la temperatura, humedad relativa y velocidad del viento. Las gráficas presentadas en este trabajo se realizaron utilizando el programa OriginPro 9.64. Para las gráficas se utilizan los valores horarios de los contaminantes $\mathrm{BC}, \mathrm{CO}$ y $\mathrm{CO}_{2}$. En la Tabla 1, se muestra la estadística de todo el periodo estacional estudiado (seca caliente) agrupados por días: todos los días (TD), día de semana (DS) y fin de semana(FS). 
Tabla 1. Estadística estacional del $\mathrm{BC}$, $\mathrm{CO}$ y $\mathrm{CO}_{2}$, durante la temporada seca caliente para todos los días, día de semana (día laborable) y fin de semana, en Querétaro, México.

\begin{tabular}{lrrrrrr}
\hline Contaminante & Días & Media & D.E & Mínimo & Mediana & Máximo \\
\hline \multirow{3}{*}{$\mathrm{BC}$} & TD & 1.15 & 0.81 & 0.00 & 0.91 & 6.92 \\
& DS & 1.22 & 0.86 & 0.06 & 0.96 & 6.92 \\
& FS & 1.00 & 0.67 & 0.00 & 0.78 & 5.27 \\
\hline \multirow{2}{*}{$\mathrm{CO}$} & TD & 0.28 & 0.17 & 0.11 & 0.23 & 1.73 \\
& DS & 0.29 & 0.19 & 0.11 & 0.23 & 1.73 \\
& FS & 0.26 & 0.12 & 0.13 & 0.22 & 0.76 \\
\hline \multirow{2}{*}{$\mathrm{CO}_{2}$} & TD & 406.26 & 8.08 & 395.31 & 403.75 & 451.15 \\
& DS & 406.67 & 8.75 & 395.45 & 403.92 & 451.15 \\
& FS & 405.21 & 5.88 & 395.31 & 403.32 & 431.61 \\
\hline
\end{tabular}

$\mathrm{TD}=$ todos los días, $\mathrm{DS}=$ día de semana, $\mathrm{FS}=$ Fin de semana, $\mathrm{DE}=$ desviación estándar

\section{RESULTADOS}

La Figura 1a-c muestra los gráficos de caja y bigotes, utilizados para representar la distribución de las mediciones del $\mathrm{BC}, \mathrm{CO}$ y $\mathrm{CO}_{2}$, durante marzo, abril y la temporada seca caliente, respectivamente. En estos gráficos, el círculo y la línea horizontal en el interior de la caja representan el valor medio y la mediana, respectivamente. El fondo de la caja representa al cuartil $25 \%$; y su tope, al cuartil $75 \%$.
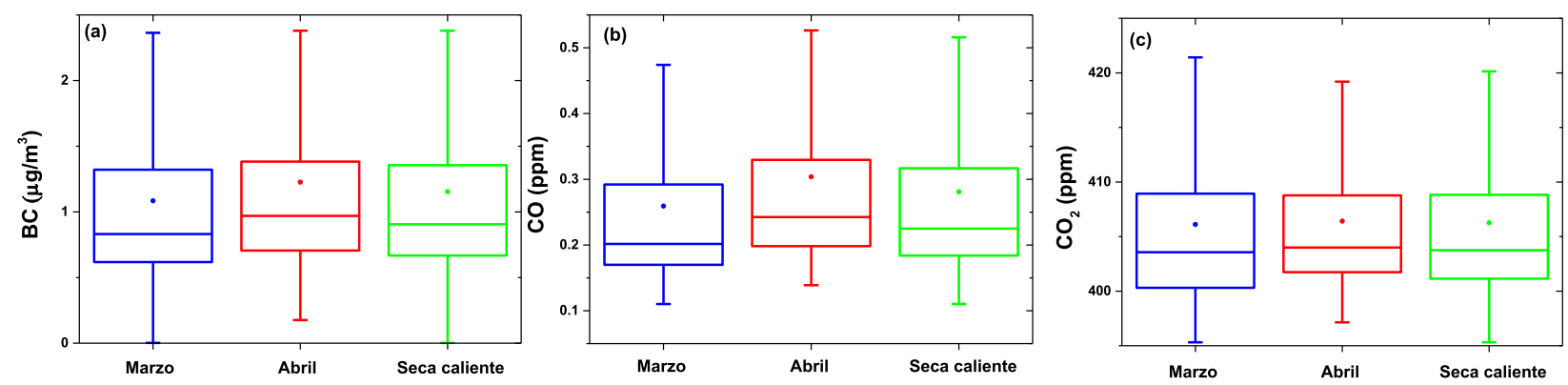

Figura 1. Gráfico de caja y bigotes, para representar la distribución de los valores horarios del (a) BC, (b) $\mathrm{CO}$ y (c) $\mathrm{CO}_{2}$ en Querétaro, México.

La Figura 2a-c muestra la distribución, mediante gráficos de caja y bigotes, de las mediciones de los parámetros meteorológicos (temperatura, humedad relativa y velocidad del viento), durante marzo, abril y la temporada seca caliente, respectivamente. Es conocida la anticorrelación entre la humedad relativa y la temperatura, lo que se evidencia en estos gráficos. Cabe destacar la menor temperatura y la relativamente mayor rapidez del viento en marzo, comparados con sus valores correspondientes al mes de abril. El valor promedio de la velocidad del viento en el mes de marzo fue de $3.25 \mathrm{~m} / \mathrm{s} ;$ mientras que, en abril fue de $2.65 \mathrm{~m} / \mathrm{s}$. La temperatura promedio en abril fue $21.24^{\circ} \mathrm{C}$, y en marzo aproximadamente $3^{\circ} \mathrm{C}$ menor $\left(18.12{ }^{\circ} \mathrm{C}\right)$. Ambos parámetros son importantes para hacer una evaluación cualitativa del desarrollo de la capa de mezcla atmosférica. 

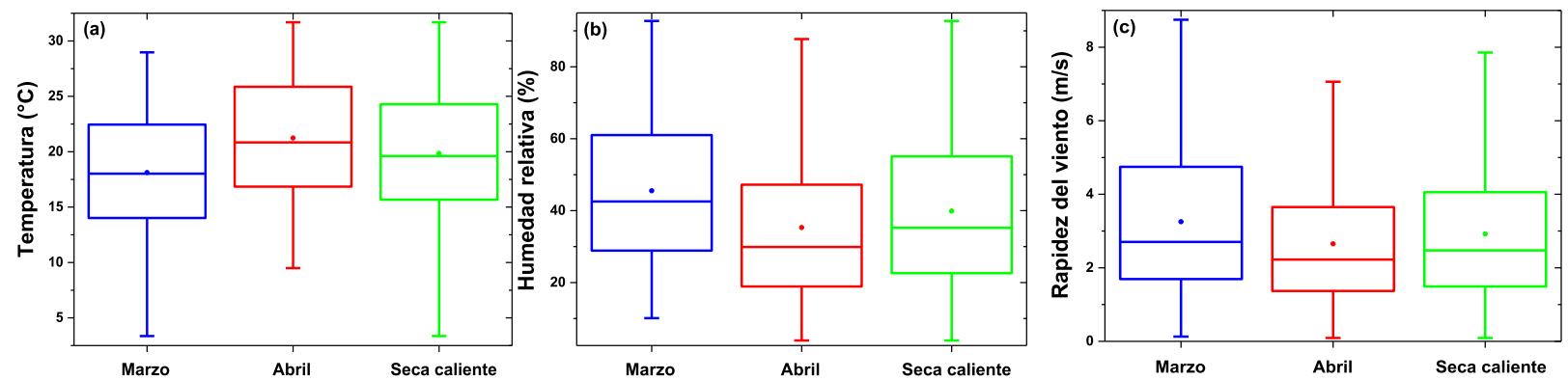

Figura 2. Gráfico de caja y bigotes para representar la distribución de los valores de (a) Temperatura, (b) Humedad relativa y (c) rapidez del tiempo en Querétaro, México.

\section{Serie de tiempo de concentraciones de $\mathrm{BC}, \mathrm{CO} \mathrm{y} \mathrm{Co}_{2}$}

La Figura 3 muestra las series de tiempo de los valores horarios de $\mathrm{BC}, \mathrm{CO}_{\mathrm{CO}_{2}}$ en Querétaro, desde el 01 de marzo al 30 de abril del 2016. Los valores de $\mathrm{BC}, \mathrm{CO}$ y $\mathrm{CO}_{2}$ mostraron variaciones temporales correlacionadas. La estadística de estos contaminantes, durante la temporada seca caliente, se resume en la Tabla 1. Durante la estación seca caliente, la concentración en masa del BC osciló entre 0.00 y $6.92 \mu \mathrm{g} / \mathrm{m}^{3}$, con un valor promedio de $1.15 \mu \mathrm{g} / \mathrm{m}^{3}$. La relación de mezcla del CO osciló entre 0.11 y 1.73 partes por millón por volumen (ppmv o ppm), con un valor medio de $0.28 \mathrm{ppm}$; mientas que la del $\mathrm{CO}_{2}$ tuvo una variación de 395.31 hasta $451.15 \mathrm{ppm}$, obteniendo un valor promedio de $406.26 \mathrm{ppm}$.

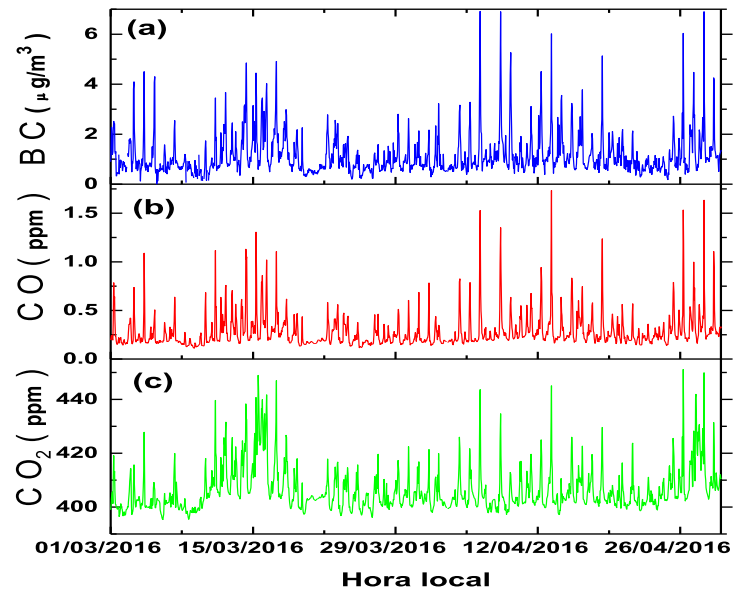

Figura 3. Serie de tiempo de las concentraciones de: (a) BC, (b) $\mathrm{CO}$ y (c) $\mathrm{CO}_{2}$, del 01 de marzo al 30 de abril del 2016 en Querétaro, México.

Las variaciones mensuales de $\mathrm{BC}, \mathrm{CO}$ y $\mathrm{CO}_{2}$ en Querétaro son evidentes (Tabla 1 y Figura 3). El valor promedio de la concentración de $\mathrm{BC}$ en abril $\left(1.23 \mu \mathrm{g} / \mathrm{m}^{3}\right)$ es mayor al valor de $\mathrm{BC}$, correspondiente a marzo $\left(1.08 \mu \mathrm{g} / \mathrm{m}^{3}\right)$. Similarmente, el valor promedio de la relación de mezcla del CO en abril (0.30 ppm) es mayor que en marzo ( $0.26 \mathrm{ppm})$; mientras que el $\mathrm{CO}_{2}$ no sufre variación en estos meses y mantiene un valor que esta alrededor de $406 \mathrm{ppm}$.

\section{Ciclos diarios de $\mathrm{BC},{\mathrm{CO} \mathrm{y} \mathrm{CO}_{2}}_{2}$}

En la Figura 4(a-c) se muestran las tendencias diarias de la concentración en masa del carbono negro (BC), y las relaciones de mezcla del monóxido de carbono (CO) y el dióxido de carbono $\left(\mathrm{CO}_{2}\right)$, durante la estación seca caliente en Querétaro. El ciclo diario del BC presenta un máximo pronunciado por la mañana y un mínimo por la tarde (Figura 4.a). El BC empieza a incrementarse rápidamente, aproximadamente desde las 06:00-07:00 HL, hasta las 08:00-09:00 $\mathrm{HL}$, tiempo en el que alcanza su valor horario máximo de $2.55 \mu \mathrm{g} / \mathrm{m}^{3}$. Luego, el BC cae hasta alcanzar su mínimo por la tarde, alrededor de las 15:00-16:00 HL.

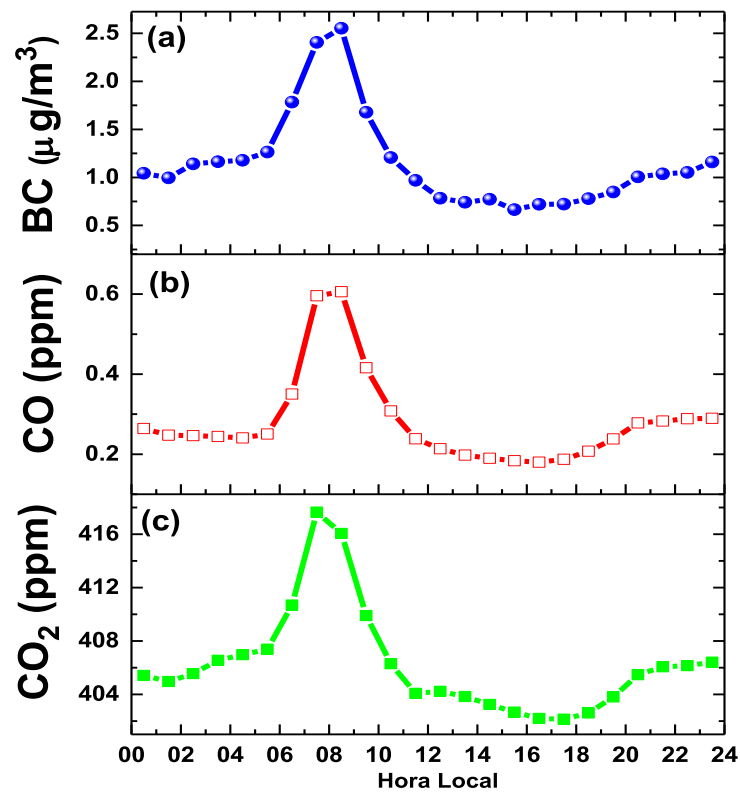

Figura 4. Ciclo diario de las concentraciones de (a) BC, (b) $\mathrm{CO}$ y (c) $\mathrm{CO}_{2}$, durante la temporada seca caliente del 2016 en Querétaro, México. 
La Figura 4.b muestra la variación diaria estacional del CO. Los ciclos diarios del CO son muy similares a los del $\mathrm{BC}$, dado que, bajo las mismas condiciones meteorológicas, ambos podrían tener las mismas fuentes. La variación diaria del $\mathrm{CO}_{2}$ en Querétaro se muestra en la Figura 4.c. El ciclo diario estacional del $\mathrm{CO}_{2}$ presenta dos máximos (temprano en la mañana y en la noche) y un mínimo (en la tarde). El primer máximo lo alcanza entre las 07:00-08:00 HL, probablemente se deba al incremento de las emisiones vehiculares.

\section{Correlaciones: $\mathrm{BC}-\mathrm{CO}, \mathrm{BC}-\mathrm{CO}_{2}$ y CO-CO}

En este trabajo se realizaron las siguientes correlaciones: $\mathrm{BC}-\mathrm{CO}, \mathrm{BC}-\mathrm{CO}_{2}$ y $\mathrm{CO}-\mathrm{CO}_{2}$, con el fin de estimar las fuentes importantes de $\mathrm{BC}$ y $\mathrm{CO}$ a partir de las pendientes de estas correlaciones, y poder establecer una metodología que permita estimar las relaciones de emisión $\mathrm{BC} / \mathrm{CO}, \mathrm{BC} / \mathrm{CO}_{2} \mathrm{y}$ $\mathrm{CO} / \mathrm{CO}_{2}$.
La Figura 5 muestra los diagramas de dispersión de (a) BC-CO, (b) $\mathrm{BC}-\mathrm{CO}_{2}$ y (c) $\mathrm{CO}-\mathrm{CO}_{2}$, para la estación seca caliente en Querétaro. Las pendientes de las rectas en los diagramas: $\mathrm{BC}-\mathrm{CO}$ $(\Delta \mathrm{BC} / \Delta \mathrm{CO}), \mathrm{BC}-\mathrm{CO}_{2}\left(\Delta \mathrm{BC} / \Delta \mathrm{CO}_{2}\right)$ y $\mathrm{CO}-\mathrm{CO}_{2}$ $\left(\Delta \mathrm{CO} / \Delta \mathrm{CO}_{2}\right)$ se obtuvieron mediante el método de mínimos cuadrados. EL BC correlaciona muy bien con $\mathrm{CO}$, con un coeficiente de determinación $\left(\mathrm{R}^{2}\right)$ de 0.79 y una pendiente $\triangle \mathrm{BC} / \Delta \mathrm{CO}=4.18$ $\mu \mathrm{gm}^{-3} / \mathrm{ppm}$, lo cual se puede observar en la Figura 5.a. La Figura 5.b muestra la gráfica de dispersión $\mathrm{BC}-\mathrm{CO}_{2}$. El $\mathrm{R}^{2}$ del ajuste lineal es 0.56 . La pendiente $\Delta \mathrm{BC} / \Delta \mathrm{CO}_{2}=0.08 \mu \mathrm{gm}^{-3} / \mathrm{ppm}$, en la estación seca caliente. En la Figura 5.c se muestra la gráfica de dispersión $\mathrm{CO}-\mathrm{CO}_{2}$ en la estación seca caliente. La correlación es buena, con un $\mathrm{R}^{2}$ de 0.64 y una pendiente $\Delta \mathrm{CO} / \Delta \mathrm{CO}_{2}=0.02$ $\mathrm{ppm} / \mathrm{ppm}$. En general, las pendientes $\Delta \mathrm{BC} / \Delta \mathrm{CO}$, $\Delta \mathrm{BC} / \Delta \mathrm{CO}_{2} \mathrm{y} \Delta \mathrm{CO} / \Delta \mathrm{CO}_{2}$ correlacionan bien.
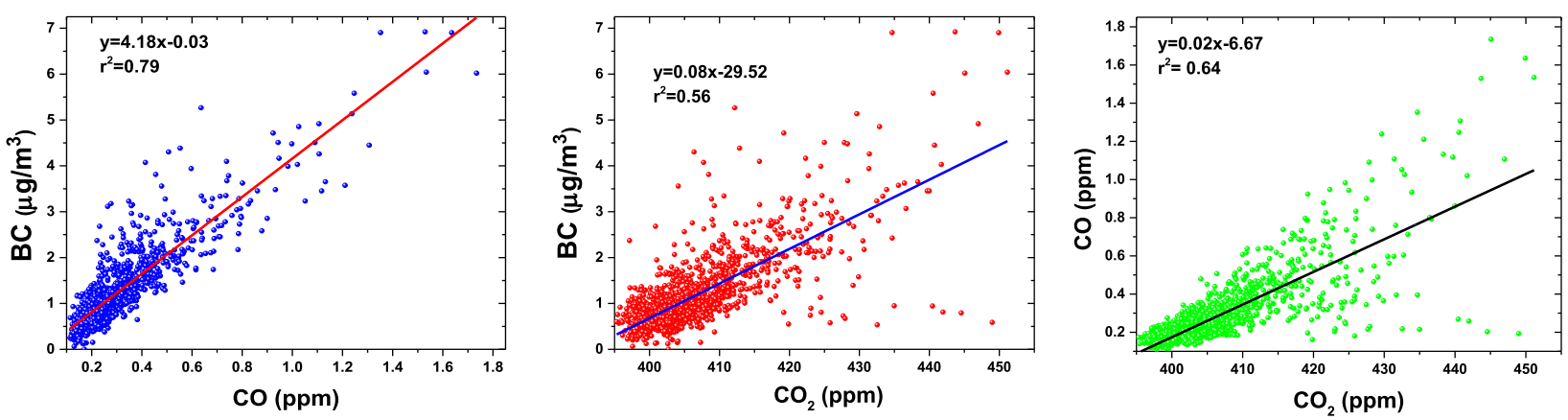

Figura 5. Gráficas de dispersión de (a) $\mathrm{BC}-\mathrm{CO}$, (b) $\mathrm{BC}-\mathrm{CO}_{2}$ y (c) $\mathrm{CO}-\mathrm{CO}_{2}$, para la temporada seca caliente, en Querétaro, México. Se muestra la ecuación de la recta obtenida mediante ajuste lineal y el coeficiente de determinación $\left(r^{2}\right)$.

\section{El efecto fin de semana}

El efecto fin de semana de $\mathrm{BC}, \mathrm{CO}$ y $\mathrm{CO}_{2}$ se caracteriza por las bajas concentraciones de estos contaminantes en fines de semana comparadas con las de días de semana, debido a una disminución en las actividades antropogénicas (disminución de la actividad laboral y de las instituciones educativas) y a una menor densidad de tráfico vehicular durante el fin de semana. La Tabla 1 muestra las concentraciones promedio del $\mathrm{BC}, \mathrm{CO}$ y $\mathrm{CO}_{2}$, junto a su respectiva desviación estándar, para los días de semana y fines de semana. El valor promedio de la concentración de $\mathrm{BC}$ decrece en $18 \%$ los fines de semana (de 1.22 $\mu \mathrm{g} / \mathrm{m}^{3}$ a $\left.1.00 \mu \mathrm{g} / \mathrm{m}^{3}\right)$. La concentración promedio del CO de un día laborable a un día de fin de semana cae aproximadamente $10 \%$ (de $0.29 \mathrm{ppm}$ a $0.26 \mathrm{ppm}$ ); mientras que el valor promedio de la concentración del $\mathrm{CO}_{2}$ se reduce menos del $1 \%$ (de 406.67 ppm a 405.21 ppm). La disminución de los valores máximos de las concentraciones entre un día de semana y fin de semana son aproximadamente $24 \%, 56 \%$ y $4 \%$ para el BC, $\mathrm{CO}$ y $\mathrm{CO}_{2}$, respectivamente. La Figura 6 muestra las variaciones diarias de las concentraciones de (a) $\mathrm{BC}$, (b) $\mathrm{CO}$ y (c) $\mathrm{CO}_{2}$, para un día de semana y un día de fin de semana (sábado y domingo) en Querétaro. Las tendencias diarias de estos contaminantes para un día laborable son similares a sus ciclos diarios para todos los días mostrados en la Figura 5. 


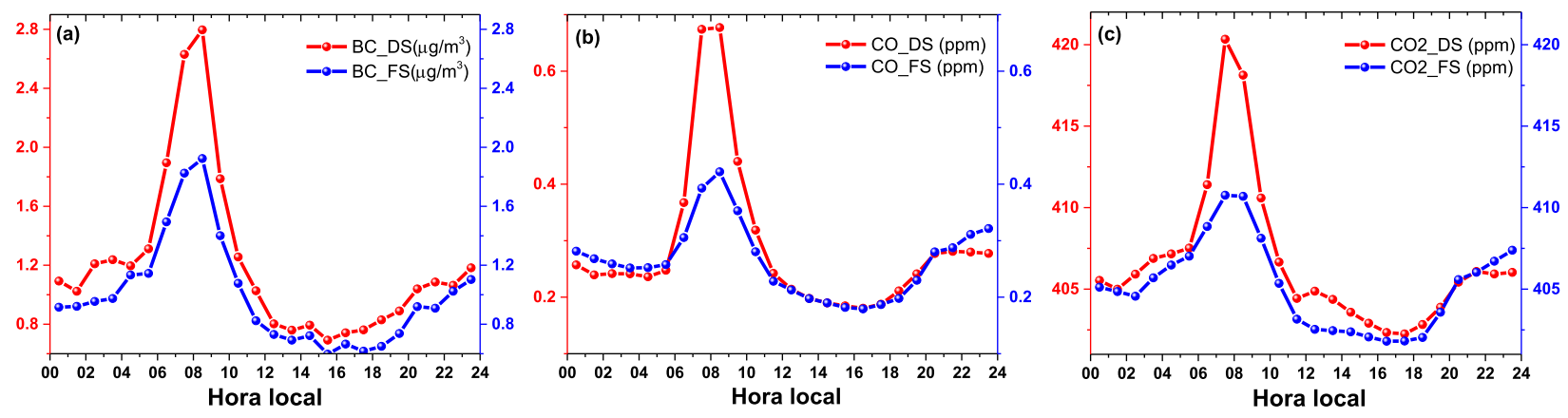

Figura 6. Ciclo diario de las concentraciones de (a) $\mathrm{BC}$, (b) $\mathrm{CO}$ y (c) $\mathrm{CO}_{2}$, para días de semana (DS) y días de fin de semana (FS) en Querétaro, México.

\section{DISCUSIÓN}

En Querétaro, los valores del $\mathrm{BC}$ y $\mathrm{CO}$ son mayores en el mes de abril que en marzo (Figura 3). Este comportamiento mensual se debería al mayor valor promedio de la rapidez del viento en marzo $(3.25 \mathrm{~m} / \mathrm{s})$, que en abril $(2.65 \mathrm{~m} / \mathrm{s})$. A mayor ventilación, menor es la concentración de contaminantes. Mientras que el valor promedio del $\mathrm{CO}_{2}$ no sufre variación en estos meses y mantiene un valor que está alrededor de $406 \mathrm{ppm}$. Es conocido que las concentraciones absolutas de $\mathrm{BC}, \mathrm{CO}$ y $\mathrm{CO}_{2}$ presentan una fuerte dependencia de las emisiones y las condiciones meteorológicas (Han et al., 2009; Zhuang et al, 2014; Zhang et al., 2015).

El patrón diario estacional del $\mathrm{BC}$ se puede explicar mediante la combinación de los dos factores que afectan su concentración: emisiones y condiciones meteorológicas. El incremento de la concentración del BC, que se inicia en horas de la madrugada hasta alcanzar su valor máximo diario (08:00-09:00 HL) coincide con el inicio de las actividades antropogénicas, como la circulación del transporte público y privado en la ciudad. La posterior caída del BC, desde su máximo hasta su mínimo, coincide con el crecimiento diurno de la profundidad de la capa de mezcla (capa de la atmósfera adyacente al suelo donde los contaminantes emitidos son dispersados por convección o turbulencia), desde su valor mínimo hasta su máxima profundidad, que alcanza por las tardes, momento donde se dispone de un mayor volumen de aire que facilita la dispersión de los contaminantes.

El segundo aumento de la concentración de BC hasta la noche estaría relacionado con la acumulación más eficiente de $\mathrm{BC}$ en la atmosfera, debido a una disminución de la altura de la capa de mezcla y la velocidad del viento. Los ciclos diarios del CO son muy similares a los del BC. La concentración de $\mathrm{CO}$ depende fuertemente de las emisiones de los vehículos ligeros a gasolina (LDGVs) y el BC de los vehículos pesados a diésel (HDDVs). El ciclo diario estacional del $\mathrm{CO}_{2}$ presenta una tendencia similar a las del $\mathrm{BC}$ y CO. Después de la salida del sol, la absorción fotosintética de $\mathrm{CO}_{2}$ por la vegetación y el desarrollo de la altura de la capa de mezcla hacen que las concentraciones de $\mathrm{CO}_{2}$ disminuyan gradualmente y alcancen un mínimo estable por la tarde. Por la noche, cuando domina la respiración y la capa de mezcla disminuye, el $\mathrm{CO}_{2}$ se incrementa y alcanza un segundo máximo.

La buena correlación observada entre sí, por estos contaminantes (Figura 5), sugiere que los contaminantes provendrían de fuentes móviles (transporte urbano). Estudios previos en áreas urbanas, demuestran que $\mathrm{BC}$ correlaciona muy bien con el CO, debido a que ambos contaminantes primarios son emitidos durante la combustión incompleta de combustibles basados en carbono, especialmente combustibles fósiles (Baumgardner et al., 2002; Kondo et al., 2006; Han et al., 2009). Las correlaciones entre BC y CO dependen principalmente del tipo de fuente de emisión, aun cuando se encuentren en la misma región (Bond et al., 2006). Si BC y CO provienen de la misma fuente se esperan altas correlaciones (Zhuang et al., 2012).

Los valores promedios de las concentraciones de $\mathrm{BC}$ y $\mathrm{CO}$ presentan un notable efecto fin de semana; mientras que la concentración promedio del $\mathrm{CO}_{2}$ no cambia notablemente de un día de semana a uno de fin de semana. Las tendencias diarias para un día de fin de semana presentan 
máximos menos agudos y simultáneos con los de un día de semana. Además, se observa que las concentraciones de $\mathrm{CO}$, durante la noche y las madrugadas de los fines de semana son mayores a los valores del CO durante los días laborables o día de semana. Estos resultados indican que la variación diaria de la actividad antropogénica fue diferente entre los días laborables y los fines de semana. De hecho, el tráfico (fuente más importante de emisiones primarias de $\mathrm{BC}$ y $\mathrm{CO}, \mathrm{y}$ menor medida de $\mathrm{CO}_{2}$ ) presenta patrones muy diferentes durante los días de semana y fines de semana. En general, en todo el ciclo diario, el tráfico de vehículos ligeros a gasolina (LDGVs) y vehículos pesados a diésel (HDDVs) es menor los fines de semana, excepto durante la madrugada, donde el tráfico de los LDGVs es mucho mayor, lo que explica que las concentraciones de $\mathrm{CO}$ de un día de fin de semana sean superiores a los de un día de semana en las madrugadas.

\section{CONCLUSIONES}

Durante la estación seca caliente, el valor promedio de la concentración de BC en abril (1.23 $\mu \mathrm{g} / \mathrm{m}^{3}$ ) es mayor al valor de BC correspondiente a marzo $\left(1.08 \mu \mathrm{g} / \mathrm{m}^{3}\right)$. Similarmente, el valor promedio de la relación de mezcla del $\mathrm{CO}$ en abril $(0.30 \mathrm{ppm})$ es mayor que marzo $(0.26 \mathrm{ppm})$; mientras que el $\mathrm{CO}_{2}$ no sufre variación en estos meses y mantiene un valor que esta alrededor de $406 \mathrm{ppm}$.

Probablemente, la razón de los mayores valores de estos contaminantes en abril sea por el mayor valor promedio de la rapidez del viento en marzo $(3.25 \mathrm{~m} / \mathrm{s})$ que en abril $(2.65 \mathrm{~m} / \mathrm{s})$. A mayor ventilación menor es la concentración de contaminantes.

Los ciclos diarios estacionales del $\mathrm{BC}, \mathrm{CO}$ y $\mathrm{CO}_{2}$ presentan un máximo pronunciado por la mañana y un mínimo por la tarde. Empiezan a incrementarse rápidamente desde las 06:00-07:00 HL hasta las 08:00-09:00 HL aproximadamente, tiempo en el que alcanzan su valor horario máximo. Luego, la tendencia cae hasta alcanzar su mínimo por la tarde, alrededor de las 15:00-16:00 HL.

En general, las correlaciones BC-CO, BC- $\mathrm{CO}_{2}, \mathrm{y}$ $\mathrm{CO}-\mathrm{CO}_{2}$ fueron buenas con valores de sus pendientes de $4.18 \mu \mathrm{gm}^{-3} / \mathrm{ppm}, 0.08 \mu \mathrm{gm}^{-3} / \mathrm{ppm}, \mathrm{y}$ $0.02 \mathrm{ppm} / \mathrm{ppm}$; respectivamente.

Estas buenas correlaciones observadas podrían deberse al hecho de que estos contaminantes provengan de las mismas fuentes, vinculadas a la combustión incompleta de combustibles basados en carbono, especialmente combustibles fósiles. Los valores promedios de las concentraciones de BC y CO presentan un notable efecto fin de semana; mientras que la concentración promedio del $\mathrm{CO}_{2}$ no cambia notablemente de un día de semana a uno de fin de semana.

Las tendencias diarias para un día de fin de semana presentan máximos menos agudos y simultáneos con los de un día de semana. El mayor decrecimiento del $\mathrm{BC}$ que el $\mathrm{CO}$ está más vinculado al decrecimiento de la combustión de combustible diésel que de gasolina.

\section{AGRADECIMIENTO}

Agradecemos a la Red Universitaria de Observatorios Atmosféricos (RUOA) de la Universidad Nacional Autónoma de MéxicoUNAM y al CONACYT, por el valioso apoyo otorgado, fundamental para realizar esta investigación.

\section{REFERENCIAS}

Arnott, W. P., Moosmüller, H., Rogers, C. F., Jin, T. \& Bruch, R. (1999). Photoacoustic spectrometer for measuring light absorption by aerosols: Instrument description. Atmos. Environ., 33, 28452852.

Arnott, W. P., Moosmüller, H. \& Walker, J. W. (2000). Nitrogen dioxide and keroseneflame soot calibration of photoacoustic instruments for measurement of light absorption by aerosols. Rev. Sci. Inst., 71(7), 4545-4552.

Ballantine, A.P., Alden, C.B., Miller, J.B., Tans, P.P. and White, J.W.C. (2012). Increase in observed net carbon dioxide uptake by land and oceans during the past 50 years. Nature, 488, 70-73.

Baumgardner, D., Raga, G., Peralta, O., Rosas, I., Castro, T., Kuhlbusch, T., John, A. \& Pezolt, A. (2012). Diagnosing black carbon trends in large urban areas using carbon monoxide measurements. J. Geophys. Res., 107 ( D 21 ), 8342 , d o i : 10 1029/2001JD000626

Bond, T.C. \& Bergstrom, R.W. (2006). Light Absorption by carbonaceous Particles: An Investigative Review. Aerosol Science and 
Technology, 40, $27-67$. doi: $10.1080 / 02786820500421521$

Bond, T.C., Doherty, S.J., Fahey, D.W., Forster, P.M., Berntsen, T., DeAngelo, B.J., Flanner, M.G., Ghan, S., Kärcher, B., Koch, D., Kinne, S., Kondo, Y., Quinn, P.K., Sarofim, M.C., Schultz, M.G., Schultz, M., Venkataraman, C., Zhang, H., Zhang, S., Bellouin, N., Guttikunda, S.K., Hopke, P.K., Jacobson, M.Z., Kaiser, J.W., Klimont, Z., Lohmann, U., Schwarz, J.P., Shindell, D., Storelvmo, T., Warren, S.G. \& Zender, C.S. (2013). Bounding the role of black carbon in the climate system: A scientific assessment. Journal Geophysical Research: Atmospheres, 118, 5380-5552. doi: 10.1002/jgrd.50171

Bravo, J.L., Azpra, E., Zarraluqui, V. \& Gay, C. (2014). Some variations of the rainfall in Mexico City from 1954 to 1988 and their statistical significance, Atmósfera, 27(4), $367-376$ ). d o i : 10.20937/ATM.2014.27.04.03

Chen, H., Winderlich, J., Gerbig, C., Hoefer, A., Rella, C. W., Crosson, E. R., Van Pelt, A. D., Steinbach, J., Kolle, O., Beck, V., Daube, B. C., Gottlieb, E. W., Chow, V. Y., Santoni, G. W. \& Wofsy, S. C. (2010). High-accuracy continuous airborne measurements of greenhouse gases $(\mathrm{CO} 2$ and $\mathrm{CH} 4$ ) using the cavity ring-down spectroscopy (CRDS) technique. Atmos. Meas. Tech., 3, 375-386, doi:10.5194/amt3-375-2010

Crosson, E. R. (2008). A cavity ring-down analyzer for measuring atmospheric levels of methane, carbon dioxide, and water vapor. Appl. Phys. B, 92, 403-408, doi:10.1007/s00340-008-3135-y

De Foy B., Caetano, E., Magaña, V., Zitácuaro, A., Cárdenas, B., Retama, A., Ramos, R., Molina, L. \& Molina, M. (2005). Mexico City basin wind circulation during the MCMA-2003 field campaign. Atmos. Chem. Phys. 5, 2267-228. doi: 10.5194/acp-5-2267-2005

Finlayson-Pitts, B. \& Pitts, J. (2000). Chemistry of the Upper and Lower Atmosphere: Theory, Experiments and Applications, First Edition; Academic Press,.

Han, S., Kondo, Y., Oshima, N., Takegawa, N., Miyazaki, Y., Hu, M., Lin, P., Deng, Z., Zhao, Y., Sugimoto, N. \& Wu, Y. (2009).
Temporal variations of elemental carbon in Beijing, J. Geophys. Res., 114, D23202, doi: 10.1029/2009JD012027.

INEGI. (2018). Instituto Nacional de Estadística Geografía e Historia. Retrieved january 18 , 2018 , f r o m http://www.inegi.org.mx/default.aspx

IPCC (Intergovernmental Panel on Climate Change). (2013). Clouds and aerosols In: Climate Change 2013: The Physical Science Basis. Contribution of Working Group I to the Fifth Assessment Report of the Intergovernmental Panel on Climate Change. Cambridge University Press, Cambridge; United Kingdom and New York,

Jáuregui, E. (2002). The climate of the Mexico City Air Basin: its effects on the formation and transport of pollutants. In: Fenn, Mark E., Bauer, L.I. de, Hernandez-Tejeda, Tomas (Eds.), Ecological studies. Urban Air Pollution and Forest, vol. 56. Springer, New York, pp. 86-117. Chapter 5. doi: 10.1007/978-0-387-22520-3 5.

Kondo, Y., Komazaki, Y., Miyazaki, Y., Moteki, N., Takegawa, N., Kodama, D., Deguchi, S., Nogami, M., Fukuda, M., Miyakawa, T., Morino, Y., Koike, M., Sakurai, H. \& Ehara, K. (2006). Temporal variations of elemental carbon in Tokyo. J. Geophys. $R e s ., \quad 111$, D 12205 . doi:10.1029/2005JD006257.

Olivares, S. (2016). Estudio de la calidad del aire de la ciudad de Querétaro para el año 2013 (Tesis de pregrado). Universidad Nacional Autónoma de México-UNAM, Querétaro.

Seinfeld, J. \& Pandis, S. (2006). Atmospheric chemistry and physics: From Air Pollution to Climate Change, Second Edition; John Wiley \& Sons, Inc.

Zhang, X., Rao, R., Huang, Y., Mao, M., Berg, M.J. \& Sun. W. (2015). Black carbon aerosols in urban central China. Journal of Quantitative Spectroscopy \& Radiative Transfer., $150,3-11, \mathrm{doi}$ : 10.1016/j.jqsrt.2014.03.006

Zhuang, B. L., Wang, T.J., Liu, J., Li, S., Xie, M., Yang, X.Q., Fu, C.B., Sun, J.N., Yin, C.Q., Liao, J.B., Zhu, J.L. \& Zhang, Y. (2014). Continuous measurement of black carbon aerosol in urban Nanjing of Yangtze River Delta, China. Atmos. Environ., 89, 415 424, doi:10.1016/j.atmosenv.2014.02.052. 